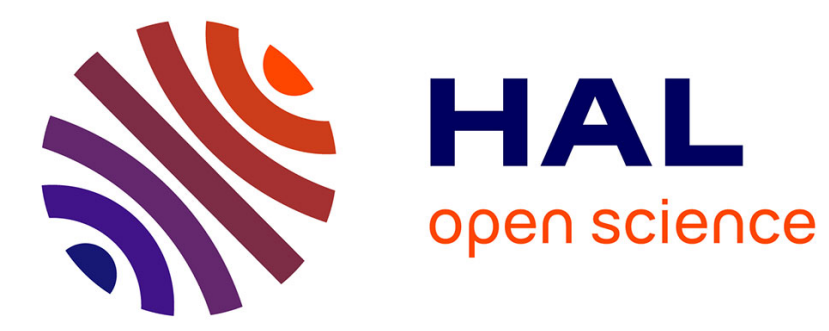

\title{
Improvement of multisensor fusion in speed limit determination by quantifying navigation reliability
}

Anne-Sophie Puthon, Fawzi Nashashibi, Benazouz Bradai

\section{To cite this version:}

Anne-Sophie Puthon, Fawzi Nashashibi, Benazouz Bradai. Improvement of multisensor fusion in speed limit determination by quantifying navigation reliability. Intelligent Transportation System Conference - ITSC 10, Sep 2010, Funchal, Portugal. pp.855 - 860, 10.1109/ITSC.2010.5625242. hal-00546167

HAL Id: hal-00546167

https://hal-mines-paristech.archives-ouvertes.fr/hal-00546167

Submitted on 13 Dec 2010

HAL is a multi-disciplinary open access archive for the deposit and dissemination of scientific research documents, whether they are published or not. The documents may come from teaching and research institutions in France or abroad, or from public or private research centers.
L'archive ouverte pluridisciplinaire HAL, est destinée au dépôt et à la diffusion de documents scientifiques de niveau recherche, publiés ou non, émanant des établissements d'enseignement et de recherche français ou étrangers, des laboratoires publics ou privés. 


\title{
Improvement of multisensor fusion in speed limit determination by quantifying navigation reliability
}

\author{
Anne-Sophie Puthon, Fawzi Nashashibi and Benazouz Bradai
}

\begin{abstract}
Speed limit determination is a complex task that may be solved by fusing data from GIS (Geographical Information System) and camera sensor. Among the existing data fusion models the Dempster-Shafer Belief Theory is found to be the most appropriate in this application. A confidence measure weights each source output, namely speed limit present on road sign and driving situation. Using the discounting scheme of Dempster-Shafer, we propose a new way of computing the navigation confidence measure by taking into account the reliability of the GIS. Preliminary tests showed that our method achieves promising results and solves conflicts between visionand navigation-based system.
\end{abstract}

\section{INTRODUCTION}

In the last decade, more and more ADAS (Advanced Driver Assistance Systems) were developed and integrated in vehicles. The reasons of such an increase was the wish to improve the car users' safety and comfort. As revealed human failures are the main car accident causes either in danger perception and analysis or during the decision process, these embedded systems help drivers in detecting and avoiding dangerous situations. Among the numerous functionalities already implemented appear LDWS (Lane Departure Warning Systems), ACC (Autonomous Cruise Control) and TSR (Traffic Sign Recognition). The latter belong to ISA (Intelligent Speed Adaptation) modules focusing on the vehicle speed regulation.

From a technological point of view, the best systems suited to ISA are found to be the autonomous ones. They only depend on the perception sensors of the vehicle contrary to the cooperative systems requiring the entire infrastructure to be equipped with beacons and an exchange protocol to be defined. However, the implementation of more and more complex applications makes a single sensor no longer sufficient [1]. Fusion requires thus to be performed between heterogeneous sensors, in order to represent the environment as accurately and completely as possible. The main problems are then sources which are inaccurate but also uncertain and the apparition of conflicts between sources.

Manuscript received July 19, 2010.

A.-S. P. is from the Robotics Centre, of Mines ParisTech, 60 boulevard Saint-Michel F-75272 Paris Cedex 06, FRANCE (phone: (33)1-40-51-9260, email: anne-sophie.puthon@ensmp.fr)

F. N. is both from the Robotics Centre of Mines ParisTech and from INRIA, Imara Team, BP 105 F-78153 Le Chesnay Cedex, FRANCE (phone: (33)1-39-63-52-56, email: fawzi.nashashibi@ensmp.fr)

B. B. is from Valeo Driving Assistance Research Center, 34 rue Saint André F-93012 BOBIGNY Cedex, FRANCE (phone: (33)1-49-42-60-95, email: benazouz.bradai@valeo.com)
In the field of ISA, current applications tend to combine a vision and a navigation system [2]. Camera-based systems aim at detecting speed signs, generally specifying particular driving conditions in dangerous or specific areas such as highway exits or calming zones ([3],[4]). Contrasting with this dynamic information, navigation-based systems provide an a priori knowledge.

Our objectives are twofold. Information corresponding to the ongoing road are firstly expressed as a set of criteria They are then divided into two classes, separating those about the sensor reliability from those determining how much confidence each speed limit may be given assuming the driving situation. These values are the modeled in the context of a subsequent fusion. More precisely, the information of the navigation-based system, corrected by the sensor reliability knowledge is combined with data from a camera-based system in order to determine more accurately the speed limit. This is achieved through the use of Dempster-Shafer model, both in discounting and fusing.

The paper is structured in three main parts. Section 2 introduces the global system integrating camera and GPS sensor. The two systems are described as well as their respective sources of inaccuracy. Then, section 3 specifies the theoretical basis of Dempster-Shafer approach and more specifically the discounting framework. Finally, the last section describes how GPS sensor deals with input data to evaluate criteria and speed limit. Our method and its results compared to existing ones are then detailed as well as the fusion process.

\section{SPEED LIMIT DETERMINATION WITH GIS AND VISION}

\section{A. System overview}

The role of ISA systems is to assist the driver in determining the speed limit at any time. By taking inspiration of human behavior, such ITS search for relevant information with the help of their embedded sensors. Drivers generally combine two types of information they got from their environment in order to regulate their speed. At first, an a priori knowledge about speed limit specified by the Highway Code gives general rules on speed limits. Then, after having detected eventual road signs, they analyse if they are concerned by the information provided. If they do not reject the sign as irrelevant, they merge both limitations 
and choose which current speed limit applies to the vehicle.

Automatic systems try to reproduce this scheme. The implicit knowledge on speed limit is obtained thanks to a navigation system. As it mainly depends on the type of road and the driving context, it is necessary to accurately locate the vehicle and match its position with the most likely road. Concerning road signs positioned on the road side, a camera-based system detects and analyzes them. The system decides then if the limitation should be applied to the vehicle or not.

Many alternatives may be used for multisensor data. The most widespread in ISA is the decision-level fusion illustrated by Fig. 1. This method consists firstly in making a decision for each source independently. Secondly, these decisions are merged globally. Assigning a decision center to each sensor offers the advantage to possibly use asynchronous sources. Consequently, the introduction of additional sources is easy. The main drawback comes from the difficulty to take into consideration dependencies and correlations between sources.

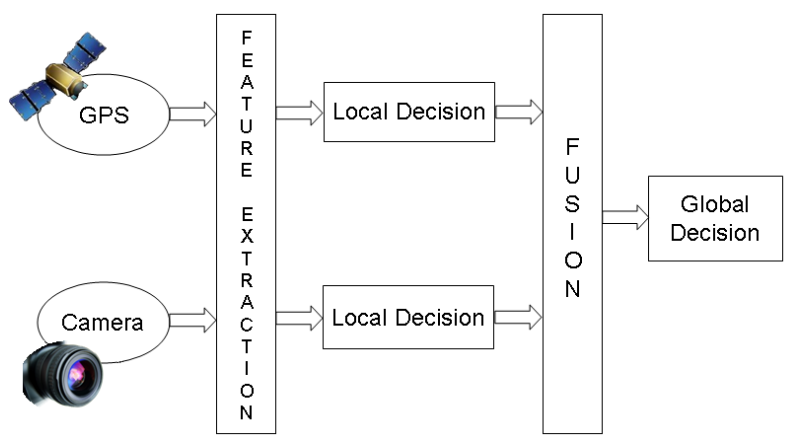

Fig. 1. Decision-level fusion model. Data of each sensor used by the application are processed by a separated decision-making unit, guaranteeing the independence. The final decision is made after the fusion of all the local decisions in order to select the most likely speed limit.

\section{B. Camera-based system}

Many studies were achieved in the field of road sign detection. The first step in TSR (Traffic Sign Recognition) is the candidate detection. It relies either on color ([5],[6]) or shape information ([7],[8]). However, since this processing does not guarantee a candidate to be an actual speed sign, recognition and classification are then used. Fulfilling the color or geometrical conditions does not guarantee candidate regions to be speed signs. They consist in selecting the right regions and determining then the value of the speed limit.

The main steps of the algorithm used in this paper are described below and illustrated by Fig. 2. The method was developed by Moutarde et al. [9] and improved by Bargeton et al. [10]. Edges are first extracted in the input grayscale image in order to apply a circular Hough transform (for circular road signs) or a rectangle detection (for American road signs for instance). Secondly in each candidate region, the bounding box of a potential number is searched. Once this step succeeded, the Region of Interest (ROI) is binarized and each digit is segmented. Then a neural network classifies the digits and irrelevant numbers are rejected. Temporal integration is performed to improve the performances of the image processing.

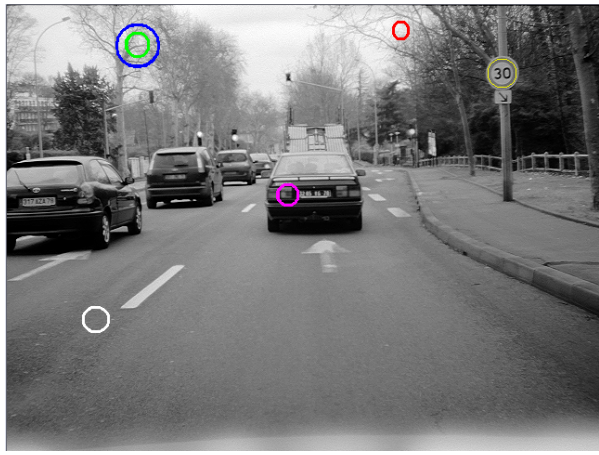

(a)

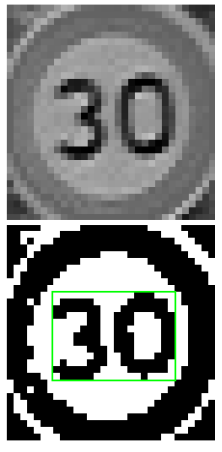

(b)
Fig. 2. After camera captured an image, a Canny filter is applied to extract edges. Circular Hough transform is then performed to search for any potential road sign in the image (a). An example of an extracted ROI is given in (b-top). The region is binarized and if a number is found its bounding box is computed (b-bottom). Each digit is then extracted and classified in order to compute the speed limit.

Despite the good results achieved with this method, some problems inherent to the use of a camera cannot be ignored. Performances of camera-based can be strongly affected by weather conditions, such as rain or fog, as underlined in [4]. Moreover, image processing may fail in detecting a speed sign because of deterioration or partial occlusion. Finally, in some situations no road sign is present and drivers thus use the Highway Code to determine the current speed limit. In our case it could be useful to use GIS data to retrieve this information.

\section{Navigation-based system}

The numerous functionalities available with the use of the GPS sensor and its low cost make it an interesting device for car manufacturers. The position output by GPS combined with digital map let us find the best path to a destination or even indicates the position of radars. The use of this sensor in determination of speed limit is then sensible. Currently, this processing relies on some attributes of the road. Fig. 3 illustrates the extraction process of such criteria. At first, a GNSS (Global Navigation Satellite-based System), like GPS, associated to a Dead-Reckoning system returns position of the vehicle. A map-matching associates this set of coordinates to the most likely road ([11],[12]). Then, an embedded digital map lists all the roads of the network. The current location is then fused with the cartography in order to select the most likely road. Finally, related attributes, previously completed by map suppliers and stored in the database, are extracted. They inform about the type of the road, driving situation or even the current speed limit. 


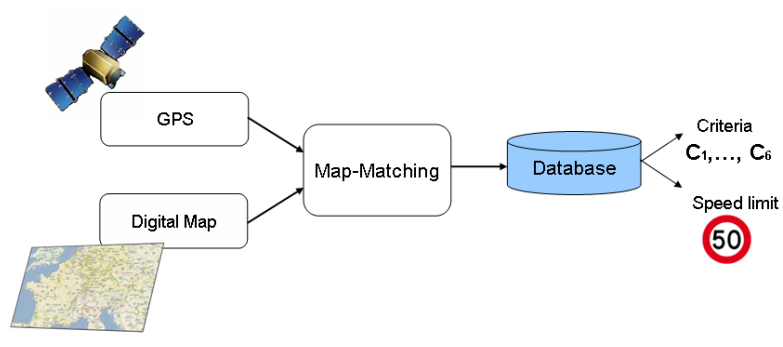

Fig. 3. Extraction of the criteria of the navigation-based system.

The navigation system presents however some limitations which need to be overcome:

- a possible wrong location of the vehicle due to a lack of visibility of satellites. Depending on its global quality a GPS accuracy interval can be up to ten meters.

- a multi-path phenomenon.

- an incorrect matching between the position and the corresponding road segment.

- the use of outdated databases particularly in urban environment, which changes often, or in roadwork.

\section{Fusion between camera and GIS}

As underlined by Nienhüser et al. [4], both vision and GPS were proved to be inaccurate under some circumstances, which can lead to erroneous evaluation. Concretely, contradictory decisions can easily be made in this case. As data may not be updated frequently enough, sensor uncertainty grows in proportion to the time elapsed since the last valid data. Ignorance quantifies situations in which sensor has no information about speed limit. For instance, when no road sign is present, no information is available to the camera-based system. Among the existing fusion theories, Dempster-Shafer theory was found to be the best model for our application. The originality of this method compared to Bayesian inference or fuzzy probability is the representation of uncertainty as well as ignorance. It also deals with conflict which occurs due to sensor inaccuracies. Furthermore, our method proposes a new way of computing belief functions with the discounting scheme, by processing the data upstream of the local decision-making. We interpret the attributes in order to establish how well accurate the GPS is.

\section{THEORETICAL BASIS}

\section{A. Belief Theory}

Belief Theory, also called Evidence Theory, was introduced by Dempster [13] and mathematically formalized by Shafer [14]. It can be viewed as a generalization of the probability theory. Handled quantities measure the belief in an event, meaning that the observations are more or less compatible with each possible solution. Mathematically speaking, the degree of belief in an event is given by a mass function which respects some constraints allowing to combine effectively knowledge from different sources.
The frame of discernment $D$ includes all $N$ hypotheses $d_{i}$, potential solution of a given problem.

$$
D=\left\{d_{1}, \ldots, d_{N}\right\}
$$

For ISA applications in France, all possible speed limits are

$\{5,10,20,30,45,50,60,70,80,90,100,110,120,130$, no-limit $\}$

The power set $2^{D}$ gathers all the possible disjunctions of $D$, which corresponds to the mass function referential.

$$
2^{D}=\left\{\emptyset,\left\{d_{1}\right\},\left\{d_{2}\right\},\left\{d_{1}, d_{2}\right\}, \ldots, D\right\}
$$

To each element of the power set is assigned a belief mass $m$, called Basic Belief Assignment (BBA), such that:

$$
\begin{gathered}
m: 2^{D} \rightarrow[0,1] \\
\sum_{A \subset D} m(A)=1
\end{gathered}
$$

In other words, a mass function defined for the subset $A=\left\{d_{1}, d_{2}\right\}$ represents a degree of belief in either $d_{1}$ or $d_{2}$. By assigning belief to all subsets of $D$, Dempster-Shafer theory deals with more complex situations as probabilities, only defined on singletons. A subset of $D$ verifying $m(A)>0$ is called a focal element. When the system evolves in a so called closed world, all the solutions belong to $D$, following the principle of exhaustiveness and exclusiveness.

$$
m(\emptyset)=0
$$

Using the BBA, a confidence interval is defined which contains the exact probability of an event $A$. The limits of this interval are given by two measures, the belief $\operatorname{Bel}(A)$ and the plausibility $\operatorname{Pl}(A)$. Its length measures the ignorance about $A$ and its complementary $\bar{A}$. The belief function measures how much hypotheses assume $A$ is the solution. It measures the part of the degree of belief exactly defined for the event $A$.

$$
\operatorname{Bel}(A)=\sum_{B \subset A, B \neq \emptyset} m(B)
$$

The plausibility measures the amount of belief which does not refute the hypothesis $A$. A proposition is plausible as long as we can not prove its negation.

$$
P l(A)=\sum_{B \cap A \neq \emptyset} m(B)=1-\operatorname{Bel}(\bar{A})
$$

\section{B. Multiple sources combination}

In the presence of multiple sources, Belief Theory fuse efficiently the information to get the most reliable result. The Dempster-Shafer conjunctive combination rule is an orthogonal combination which reinforces the belief in an event when sources agree and attenuates it when conflict. Such a situation occurs when part of a source is not reliable enough. To overcome this problem, a normalization step is performed to redistribute the mass assigned to complete ignorance.

$$
\left\{\begin{array}{l}
m(A)=\frac{1}{1-K} \sum_{B_{1} \cap \ldots \cap B_{m}=A} \prod_{j=1}^{m} m_{S_{j}}\left(B_{j}\right) \quad \text { if } A \neq \emptyset \\
m(\emptyset)=0
\end{array}\right.
$$


$K=\sum_{B_{1} \cap \ldots \cap B_{m}} \prod_{j=1}^{m} m_{S_{j}}\left(B_{j}\right)<1$ is the normalization factor.

The final solution is finally obtained through a decision rule. The maximum belief criteria is chosen among the different types of possible rules. It consists in selcting the decision $d^{*}$ with the highest belief value.

$$
\operatorname{Bel}\left(d^{*}\right)=\max _{1 \leq j \leq m} \operatorname{Bel}\left(d_{j}\right)
$$

\section{Discounting scheme}

Using source reliability knowledge, when available, can be highly helpful. Indeed, the less reliable the source is, the more weakened the belief function is. The formal justification was proposed by Smets [15] and generalized by Mercier et al. [16]. Given a discounting rate $\alpha \in[0,1]$, the quantity $1-\alpha$ corresponds to a degree of belief in the source reliability $R$.

$$
\left\{\begin{array}{rrr}
m(\{R\}) & = & 1-\alpha \\
m(\mathbf{R}) & = & \alpha
\end{array}\right.
$$

$\mathbf{R}=\{R, N R\}$ being the set of possible values of reliability meaning source is reliable $(R)$ or not $(N R)$.

All the information given by a non-reliable source $S$ is then assigned to the universe $D$, modeling the ignorance. The resulting BBA ${ }^{\alpha} m_{S}$ verifies:

$$
\left\{\begin{array}{l}
{ }^{\alpha} m_{S}(A)=(1-\alpha) m_{S}(A) \\
{ }^{\alpha} m_{S}(D)=(1-\alpha) m_{S}(D)+\alpha
\end{array} \quad \forall A \subset D\right.
$$

\section{IMPROVEMENT OF NAVIGATION-BASED SYSTEM}

\section{A. Criteria extraction}

From the vehicle location provided by the GNSS, the system finds at first the related road segment, thanks to map-matching. The database stores the corresponding road attributes and the current speed limit. However, the latter data can be often out of date. The former helps then in determining the right limitation. In fact, the Highway Code defines implicit speed limit, regarding types of road and environment. Criteria extracted from the database help modeling this knowledge.

- $C_{1}$ : confidence in the positioning tool related to the quality of the fusion between GPS information, odometric and inertial measures as well as map-matching.

- $C_{2}$ : quality of the road network digitization. The better the resolution of the road, the more precise attributes available.

- $C_{3}$ : functional class of the road. Important trunk roads like highways and European roads belong to class $F C_{1}$ and are finer defined in the cartography.

- $C_{4}$ : type of the road (highway, primary and secondary roads, etc.).

- $C_{5}$ : driving situation and context (urban zone, highway exit, crossing, etc.).

- $C_{6}$ : guidance mode of the GPS activated or not.

\section{B. Discounting applied to navigation}

The attribute interpretation mentioned above ensues from the mathematical model used. In the Dempster-Shafer model the criteria help determining the confidence measure for every speed limit. They obviously have two main purposes. Criterion $C_{1}$, concerning the positioning tool accuracy, and $C_{4}$, specifying the road type, do not play the same role in the evaluation of the confidence measure of the system. A distinction can thus be made between two classes of information, as shown in Fig. 4. The first group $C_{r e l}$ gathers criteria considered as indications about the reliability of the sensor, that is to say $C_{1}, C_{2}, C_{3}$ and $C_{6}$. The other one, called $C_{S L}$, effectively measures confidence in a given speed limit under certain conditions. It includes $C_{4}$ and $C_{5}$. We introduced a seventh criterion $C_{7}$ in order to reinforce the confidence in the speed limit $S L_{d}$ stored in the map database.

$$
\begin{cases}C_{7}(A)=1 & \text { when } A=S L_{d} \\ C_{7}(A)=0 & \text { otherwise }\end{cases}
$$

When no limit is specified, $C_{7}$ has the same value for all $\mathrm{N}$ possible speed limits.

$$
C_{7}(A)=\frac{1}{N} \quad \forall A \subset D
$$

Discounting seems then appropriate as the sensor reliability can be taken into account in the computation of the confidence measure.

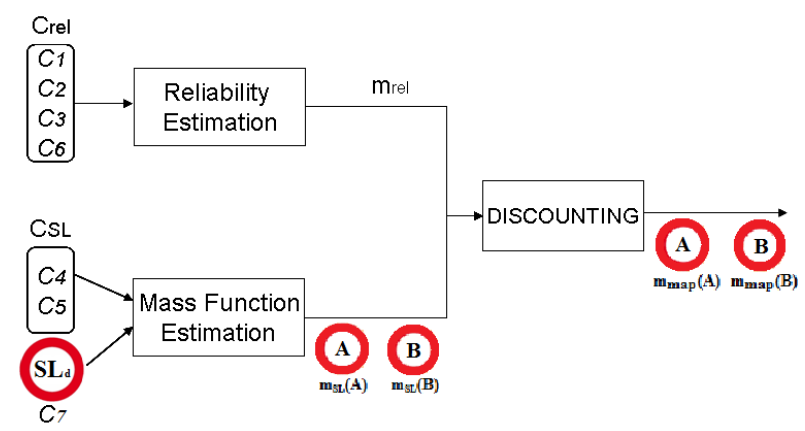

Fig. 4. Discounting scheme for the estimation of the speed limit by the navigation-based system.

In our method, the sensor reliability $m_{r e l}$ is quantified as well as the confidence for each speed limit $A$, named $m_{S L}(A)$, depending on the driving context. The final mass function $m_{\text {map }}$ is computed with the discounting method as follows:

$$
\left\{\begin{array}{ll}
m_{\text {map }}(A) & =m_{\text {rel }} m_{S L}(A) \\
m_{\text {map }}(D) & =m_{\text {rel }} m_{S L}(D)+\left(1-m_{\text {rel }}\right)
\end{array} \quad \forall A \subset D\right.
$$

Reliability is evaluated through a weighting equation using all the criteria of class $C_{r e l}$.

$$
m_{r e l}=\alpha_{1} C_{1}+\alpha_{2} C_{2}+\alpha_{3} C_{3}+\alpha_{6} C_{6}
$$

The choice of each coefficient $\alpha_{1}, \alpha_{2}, \alpha_{3}$ and $\alpha_{6}$ is based on the influence of each of them in the reliability evaluation. Since $C_{1}$ and $C_{2}$ appeared as the most influent criteria, their 
weights are the most important in the computation of $m_{\text {rel }}$, i.e. $\alpha_{1}>\alpha_{2}>\alpha_{3}>\alpha_{6}$. In order to apply the discounting scheme, $m_{r e l}$ is then normalized and thus respects the condition $\sum_{A \subset D} m_{r e l}=1$.

Both criteria $C_{4}$ and $C_{5}$ give information about the legal speed limit on a road. For instance, in the French Highway Code speed limitation on a highway is $130 \mathrm{~km} / \mathrm{h}$. The speed limit stored in the database gets a higher confidence measure through $C_{7}$.

$$
m_{S L}(A)=\alpha_{4} C_{4}+\alpha_{5} C_{5}+\alpha_{7} C_{7}
$$

As we consider importance of each criterion to be the same, $\alpha_{4}=\alpha_{5}=\alpha_{7}$.

\section{Resulting belief function}

At first, reliability $m_{\text {rel }}$ of GPS is evaluated by using (14). Assuming that $C_{1}$ and $C_{2}$ conceal most of the information about reliability, we chose $\alpha_{1}=12 / 25, \alpha_{2}=8 / 25, \alpha_{3}=$ $3 / 25$ and $\alpha_{6}=2 / 25$ (cf. Fig. 5).

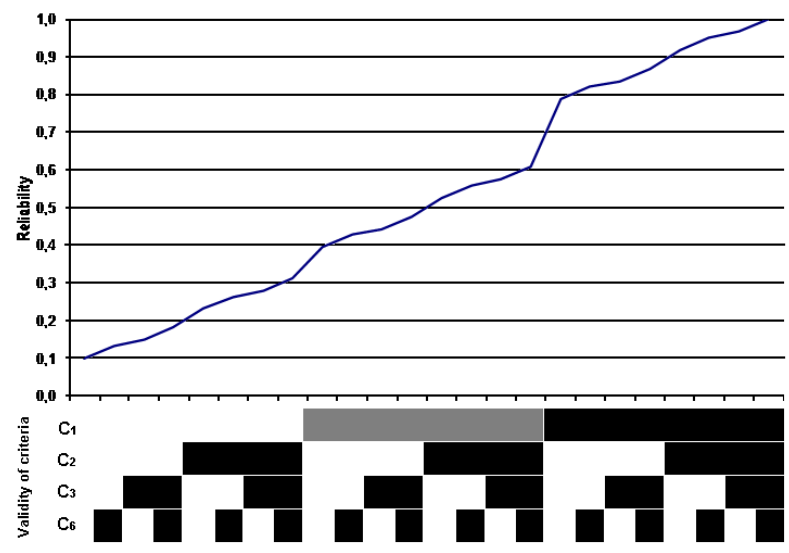

Fig. 5. Evolution of the reliability measure of the sensor depending on the validity of each criterion. The curve shape is conditioned by $C_{1}, C_{2}, C_{3}$ and $C_{6}$ values. When all criteria are validated, confidence in the evaluation of the belief function by the source is high. Practically, $m_{\text {rel }}$ is close to 1 and conversely close to 0 when dealing with an unreliable sensor.

Currently, only Lauffenburger et al. [2] implemented a method taking into account such criteria. Assuming that speed limit stored in the database can be obsolete and that GPS is sometimes inaccurate, [2] empirically defines a set of possible speed limits (focal elements) depending on the current position. BBA are then computed for each through a weighting function using all the criteria (cf. Fig. 6).

More precisely, when driving on a highway, the range of speed is expected to be around $130 \mathrm{~km} / \mathrm{h}$. This intuitive reasoning assumes that location is correct. Contrary to [2], in our method, no focal element is specified and all confidence measures depend only on the road attributes. The use of ignorance $m_{S L}(D)$ helps in distinguishing situations with reliable from those with unreliable sensors. In the case of an unreliable sensor, confidence should be weakened. Confidences in every speed limit are therefore not effectively distinguishable ( $c f$. Fig. 7).

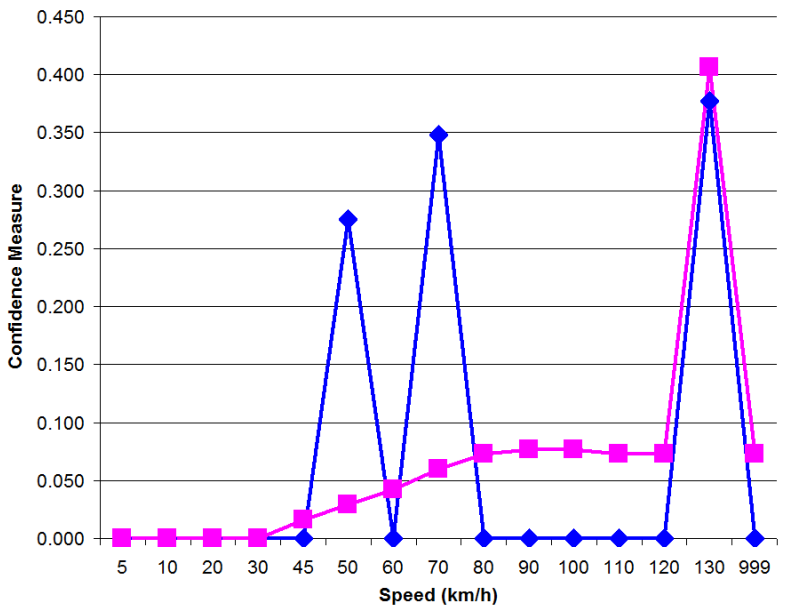

Fig. 6. Comparison between Lauffenburger method ( $\bullet$ ) and ours ( $\mathbf{\square})$. The situation corresponds to a reliable sensor, meaning that all criteria of $C_{r e l}$ are validated on a highway. The speed limit stored in the database is 130 $\mathrm{km} / \mathrm{h}$.

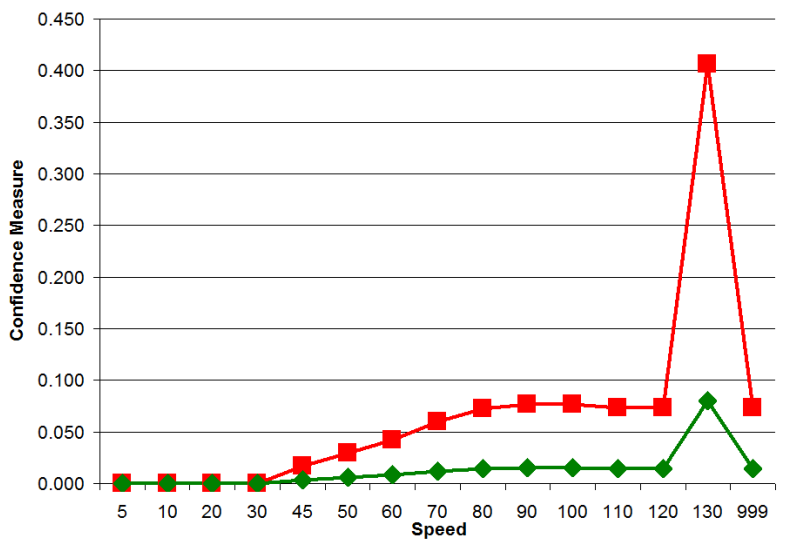

Fig. 7. Comparison of reliable ( $\square$ ) and unreliable ( $)$ sensor. The depicted case is the same situation as in Fig. 6: on a highway with a stored speed limit of $130 \mathrm{~km} / \mathrm{h}$. The shape of the curve is the same whatever is the reliability of the sensor as it only depends on the criteria of $C_{S L}$. However, the confidence values are strongly weakened when position is inaccurate and ignorance is in opposite strengthened. Ignorance is close to 0 when sensor is reliable and equal to 0.8 when unreliable.

\section{Fusion with vision}

As mentioned above, the information on speed limit provided by navigation are not perfect and do not guarantee every situation to be efficiently dealt. Therefore, a multisensor fusion is often processed in the ITS field, ideally requiring systems without any failure. By combining information from vision and navigation, our global system takes advantages of the source completeness and weakens their inaccuracies. By the use of the Dempster-Shafer conjunctive rule, sources reaching an agreement have been reinforced contrary to those in conflict. Moreover, high ignorance situations are not rejected. Fig. 8 shows an example of a driving context where the sensors are in conflict. Image processing classifies well the road sign indicating a speed limit of $110 \mathrm{~km} / \mathrm{h}$. However, the navigation-based system lacks accuracy and may locate 
the vehicle on the wrong road. Thanks to the fusion, the system determines the right speed limit.

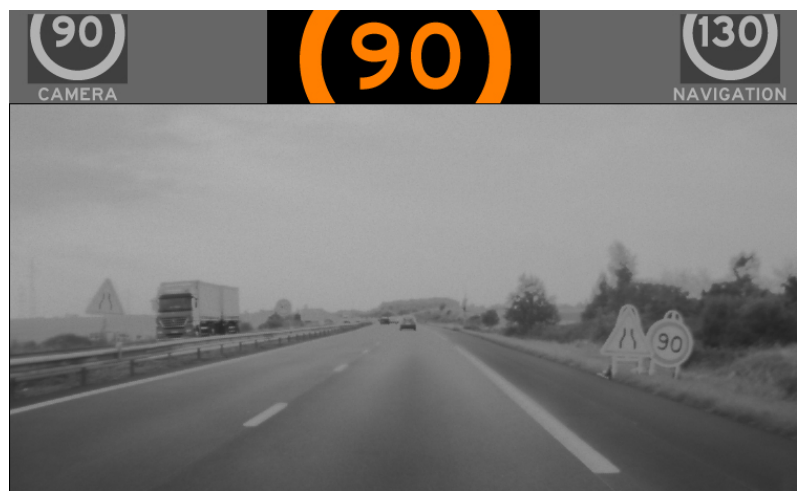

Fig. 8. Conflicting situation successfully managed with fusion. Camerabased system detected the road sign on the image and recognized a speed limit of $90 \mathrm{~km} / \mathrm{h}$ due to roadwork. On the contrary, navigation locating the vehicle on a highway output a limitation of $130 \mathrm{~km} / \mathrm{h}$. After combining the two sources of information, the decision unit chose the most likely value, which was $90 \mathrm{~km} / \mathrm{h}$.

\section{CONCLUSION}

In this paper, we showed that an inaccurate sensor such as GPS combined with a frequently out-of-date digital map, often leads to several errors. For the sake of applications using these data (such as path solving, map matching, etc.), a better accuracy would be expected. In order to do so, we introduced a new way of interpreting and processing navigation criteria. Our proposal is to separate criteria giving information about reliability from criteria dealing with driving environment. The mass function is computed from the latter. Then, reliability criteria are used to weight the mass function and also to compute the ignorance through the use of the discounting method of Dempster-Shafer.

Applying this method, let us evaluate reliability of the navigation system which is useful for a better understanding of the information it provides. Since ITS field requires high efficiency, further objectives of such an approach is the fusion of data from various sensors such as camera or radar. However for better results, integrating further information like lane marking shall be interesting.

\section{ACKNOWLEDGMENTS}

This work was conducted in the framework of the Speedcam project (ANR-09-VTT-11), funded by the ANR (Agence Nationale de la Recherche). This DEUFRAKO project gathers partners from France, which are Ecole des Mines de Paris and Valeo, but also from Germany, Daimler Benz and HTW Aalen.

\section{REFERENCES}

[1] M. Darms and H. Winner, "A Modular System Architecture for Sensor Data Processing of ADAS Applications," in Proc. of IEEE Intelligent Vehicles Symposium (IV'05), Las Vegas, USA, 2005, pp. 729-734.

[2] J. Lauffenburger, B. Bradai, M. Basset, and F. Nashashibi, "Navigation and Speed Signs Recognition Fusion for Enhanced Vehicle Location," in Proc. of International Federation of Automatic Control (IFAC'08), Seoul, Korea, 2008.

[3] O. Hamdoun, A. Bargeton, F. Moutarde, B. Bradai, L. Chanussot et al., "Detection and Recognition of End-of-Speed-Limit and Supplementary Signs for Improved European Speed Limit Support," in Proc. of World Congress on Intelligent Transport Systems (ITS'08), vol. 15, New York, USA, 2008, pp. 289-303.

[4] D. Nienhuser, T. Gumpp, and J. Zollner, "A Situation Context Aware Dempster-Shafer Fusion of Digital Maps and a Road Sign Recognition System," in Proc. of IEEE Intelligent Vehicles Symposium (IV'09), Xi' an, Shaanxi, China, 2009, pp. 1401-1406.

[5] A. De La Escalera, J. Armingol, and M. Mata, "Traffic Sign Recognition and Analysis for Intelligent Vehicles," Image and Vision Computing, vol. 21, no. 3, pp. 247-258, 2003.

[6] J. Torresen, J. Bakke, and L. Sekanina, "Efficient Recognition of Speed Limit Signs," in Proc. of IEEE Conference on Intelligent Transportation Systems (ITS'04), Maceiò, Alagoas, Brazil, 2004, pp. 652-656.

[7] N. Barnes and A. Zelinsky, "Real-time Radial Symmetry for Speed Sign Detection," in Proc. of IEEE Intelligent Vehicles Symposium (IV'04), Parma, Italy, 2004, pp. 566-571.

[8] D. Gavrila, "Traffic Sign Recognition Revisited," in Proc. of 21st DAGM-Symposium Mustererkennung. Springer-Verlag, 1999, pp. 8693.

[9] F. Moutarde, A. Bargeton, A. Herbin, and L. Chanussot, "Modular Traffic Sign Recognition Applied to On-vehicle Real-time Visual Detection of American and European Speed Limit Signs," in Proc. of World Congress on Intelligent Transportation Systems (ITS'07), Beijing, China, 2007, pp. 9-13.

[10] A. Bargeton, F. Moutarde, F. Nashashibi, and B. Bradai, "Improving Pan-European Speed-Limit Signs Recognition with a New "Global Number Segmentation" Before Digit Recognition," in Proc. of IEEE Intelligent Vehicles Symposium (IV'08), Eindhoven, Netherlands, 2008, pp. 1022-1027.

[11] Y. Zhao, Vehicle Location and Navigation Systems. Artech House Publishers, 1997.

[12] M. El Najjar and P. Bonnifait, "Road Selection Using Multicriteria Fusion for the Road-Matching Problem," IEEE Transactions on Intelligent Transportation Systems, vol. 8, pp. 279-291, 2007.

[13] A. Dempster, "Upper and Lower Probabilities Induced by a Multivalued Mapping," Classic Works of the Dempster-Shafer Theory of Belief Functions, pp. 57-72, 1967.

[14] G. Shafer, A Mathematical Theory of Evidence. Princeton university press Princeton, NJ, 1976.

[15] P. Smets, "Belief Functions: the Disjunctive Rule of Combination and the Generalized Bayesian Theorem," Classic Works of the DempsterShafer Theory of Belief Functions, pp. 633-664, 2005.

[16] D. Mercier, B. Quost, and T. Denœux, "Contextual Discounting of Belief Functions," Symbolic and Quantitative Approaches to Reasoning with Uncertainty, pp. 552-562, 2005. 